\title{
Tecnologia assistiva e a redução do estresse em cuidadores de idosos: revisão integrativa
}

\section{Assistive technology and stress reduction in caregivers of older adults: an integrative review}

\section{Tecnología asistiva y la reducción del estrés de cuidadores de mayores: revisión integrativa}

\author{
Gerarlene Ponte Guimarães Santos ii \\ Universidade Federal do Piauí - Teresina (PI) - Brasil \\ Universidade Estadual do Piauí - Parnaíba (PI) - Brasil \\ Cleidiane Maria Sales de Brito (iD \\ Universidade Federal do Piauí - Teresina (PI) - Brasil \\ Universidade Estadual do Piauí - Parnaíba (PI) - Brasil
}

Rayla Maria Pontes Guimarães Costa (iD

Universidade Federal do Piauí - Teresina (PI) - Brasil

Universidade Estadual do Piauí - Parnaíba (PI) - Brasil

Herla Maria Furtado Jorge (iD

Universidade Federal do Piauí - Teresina (PI) - Brasil

Maria do Livramento Fortes Figueiredo (iD

Universidade Federal do Piauí - Teresina (PI) - Brasil

Márcia Teles de Oliveira Gouveia iD

Universidade Federal do Piauí - Teresina (PI) - Brasil

Márcia Astrês Fernandes iD

Universidade Federal do Piauí - Teresina (PI) - Brasil

Ana Roberta Vilarouca da Silva (iD)

Universidade Federal do Piauí - Teresina (PI) - Brasil

\section{RESUMO}

Objetivo: Sintetizar as evidências científicas acerca do uso de tecnologia assistiva como estratégia para a redução do estresse em cuidadores de idosos. Métodos: Trata-se de uma revisão integrativa, sem recortes temporal, realizada no período de abril a maio de 2020 nas bases de dados: Medline/PubMed, Web of Science, Cinahl, Scopus e Lilacs/BVS, a partir da pergunta norteadora: quais as evidências do uso da tecnologia assistiva na redução do estresse em cuidadores de idosos? Para a busca, se recorreu aos descritores controlados: "aged, anciano, idoso, caregivers, cuidadores, self-help device, dispositivos de autoayuda, equipamentos de autoajuda, occupational stress, estrés laboral e estresse ocupacional", assim como os descritores não controlados e palavras-chave. Já para a extração dos dados, utilizou-se um instrumento validado e a análise descritiva. Resultados: Encontraram-se 138 artigos, sendo inclusos cinco no presente estudo. Os artigos selecionados abordam o uso das tecnologias assistivas por idosos dependentes, como redução do estresse dos seus cuidadores. São utilizados dois tipos de tecnologias assistiva, simples e complexas, a saber: bengalas, andadores, cadeiras de rodas, barras de apoio, assentos sanitários elevados, bancos de banho, robôs, programa safe home, auxiliares de memória, dispositivos de rastreamento e software de vigilância autônoma. Conclusão: As tecnologias assistivas melhoram o estresse associado à sobrecarga dos cuidadores de idosos, possibilitam melhoria para a qualidade de vida, a redução das tensões, do estresse e das frustrações.

Descritores: Equipamentos de Autoajuda; Estresse Ocupacional; Cuidadores; Idoso.

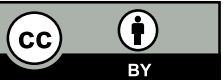




\section{ABSTRACT}

Objective: To synthesize the scientific evidence on the use of assistive technology as a strategy to reduce stress in caregivers of older adults. Methods: This is an integrative review, with no time frame, conducted from April to May 2020 in the databases: Medline/PubMed, Web of Science, Cinahl, Scopus and Lilacs/VHL, based on the research question: what is the evidence on the use of assistive technologies in reducing stress in caregivers of older adults? For the search, controlled descriptors "aged, anciano, idoso, caregivers, cuidadores, self-help device, dispositivos de autoayuda, equipamentos de autoajuda, occupational stress, estrés laboral and estresse ocupacional" and uncontrolled descriptors and keywords were used. For data extraction, a validated instrument and descriptive analysis were used. Results: Searches yielded 138 articles, five of which were included in this study. The selected articles address the use of assistive technology by dependent older adults to reduce the stress of their caregivers. Two types of assistive technologies are used, simple and complex ones, namely canes, walkers, wheelchairs, support rails, raised toilet seats, bath seats, robots, safe home program, memory aids, tracking devices and autonomous surveillance software. Conclusion: Assistive technologies improve stress associated with the work overload of caregivers of older adults, enabling improvement in quality of life, reducing tensions, stress and frustration.

Descriptors: Self-Help Devices; Occupational Stress; Caregivers; Aged.

\section{RESUMEN}

Objetivo: Sintetizar las evidencias científicas sobre el uso de la tecnología asistiva como estrategia para la reducción del estrés de cuidadores de mayores. Métodos: Se trata de una revisión integrativa, sin recortes temporales realizada en el periodo entre abril y mayo de 2020 en las bases de datos Medline/PubMed, Web of Science, Cinahl, Scopus y Lilacs/BVS, a partir de la pregunta norteadora: ¿Cuáles son las evidencias del uso de la tecnología asistiva para la reducción del estrés de cuidadores de mayores? Se ha utilizado los descriptores controlados "aged, anciano, idoso, caregivers, cuidadores, self-help device, dispositivos de autoayuda, equipamentos de autoajuda, occupational stress, estrés laboral e estresse ocupacional" para la búsqueda, así como los descriptores no controlados y palabras-clave. Para la extracción de los datos se ha utilizado un instrumento validado y el análisis descriptivo. Resultados: Se ha encontrado 138 artículos y se ha incluido cinco de ellos en ese estudio. Los artículos elegidos tratan del uso de la tecnología asistiva por mayores dependientes como de la reducción del estrés de sus cuidadores. Se utiliza dos tipos de tecnologías asistivas, simple y complejas que son: bastón, andadores ortopédicos, sillas de ruedas, barras de apoyo, asientos de baño elevados, banquillos para baño, robots, programa safe home, auxiliares de memoria, dispositivos de rastreo y software de vigilancia autónoma. Conclusión: Las tecnologías asistivas mejoran el estrés asociado con la sobrecarga de los cuidadores de mayores, posibilitan la mejoría de la calidad de vida, la reducción de las tensiones, del estrés y de las frustraciones.

Descriptores: Dispositivos de Autoayuda; Estrés Laboral; Cuidadores; Anciano.

\section{INTRODUÇÃO}

O processo de envelhecimento da população é um dos eventos mais relevantes observados no Brasil, sobretudo a partir da década de 90 , em decorrência das mudanças demográficas ocorridas a nível nacional e mundial. Nos países desenvolvidos esse processo ocorreu de forma gradual ao longo de mais de um século, já nos países em desenvolvimento e no Brasil vem ocorrendo aceleradamente ${ }^{(1)}$.

Nesse sentido, no Brasil, em 2018 as pessoas acima de 60 anos correspondiam a 15,4\% da população total, e a estimativa é de que até o ano de 2060 esse contingente populacional deverá atingir 30\%, evidenciando assim, o fenômeno do envelhecimento(2).

Indubitavelmente o número de idosos cresce a cada ano, ou seja, as pessoas estão vivendo mais, e estes anos adicionais de vida podem ocasionar declínios na capacidade física e mental, perda de massa muscular e óssea, diminuição da elasticidade dos tendões e desgastes articulares, propiciando mudanças fisiológicas que limitam ou impedem o pleno desenvolvimento das atividades de vida diária, comprometendo o autocuidado ${ }^{(3,4)}$.

Diante disso, a pessoa idosa ao experimentar essas mudanças, necessita da ajuda de cuidadores. Nesse sentido, considera-se cuidador aquela pessoa que assume a responsabilidade pelo cuidado do idoso ou de outro ser humano com algum grau de incapacidade ou dependência, podendo ser um membro da família ou não e, na maioria das vezes, desenvolve o trabalho sem formação específica voltada para o cuidado de idosos dependentes e sem remuneração(5).

Os cuidadores experimentam estresse relacionado ao trabalho desgastante que é realizado com o idoso, observado frequentemente por quadros de depressão, ansiedade, esgotamento físico e mental e piora da qualidade 
de vida ${ }^{(6)}$. Certamente, o estresse do cuidador é resultante da sobrecarga de trabalho ao lidar com a responsabilidade da prestação do cuidado ao idoso dependente, que necessita de cuidados contínuos ${ }^{(7)}$.

Nesse contexto, para auxiliar o cuidador nessa tarefa, pode-se recorrer aos recursos de Tecnologia Assistiva (TA). As tecnologias assistivas referem-se aos dispositivos, equipamentos e processos utilizados para melhoria da capacidade funcional e qualidade de vida da pessoa idosa, fornecendo resultados positivos não só aos idosos, mas também aos cuidadores ${ }^{(8,9)}$.

A utilização dos recursos de TA pode ajudar o cuidador de idosos na redução do estresse relacionado ao trabalho exercido, uma vez que, se usados de maneira correta e contínua, contribuem para a recuperação de habilidades e funções comprometidas em decorrência do processo de envelhecimento, proporcionando a pessoa idosa usufruir de um envelhecimento mais ativo, atenuando a carga de trabalho do cuidador e promovendo a saúde da díade (pessoa idosa e cuidador) ${ }^{(10)}$.

Os cuidadores também podem enfrentar dificuldades em aceitar a utilização das TA como auxílio para cuidar do outro. Em uma pesquisa internacional com cuidadores que abordavam a utilização das TA como ajuda nas atividades do cuidar, $71 \%$ afirmaram que possuíam interesse em utilizar uma TA, destes, 59\% declararam que poderiam utilizar se estivesse a sua disposição e apenas $7 \%$ usavam atualmente ou já utilizaram em atividades diárias ${ }^{(11)}$. Sugere-se, assim, que seja necessário a capacitação dos cuidadores por meio das estratégias individualizadas, para que ocorra maior adesão e, consequentemente, maior promoção à saúde do cuidador. Dessa forma, por existir uma variedade de TA, é necessário treinamento, habilidade e adesão para utilização( ${ }^{(11)}$.

Em um estudo realizado no Brasil com idosos e seus cuidadores, o uso de uma TA melhorou a comunicação terapêutica entre a díade, possibilitou modernização, disponibilidade no cuidado e promoção a saúde do cuidador ${ }^{(12)}$. Assim, faz-se necessário o presente estudo pela importância da usabilidade dos recursos de tecnologia assistiva pelas pessoas idosas, auxiliando nas tarefas diárias, contribuindo para a promoção da saúde física e mental, qualidade de vida e inclusão social, e, consequentemente, podendo diminuir a sobrecarga de trabalho dos cuidadores.

Face ao contexto supracitado, o estudo tem como objetivo sintetizar as evidências científicas acerca do uso de tecnologia assistiva como estratégia para a redução do estresse em cuidadores de idosos.

\section{MÉTODOS}

Trata-se de uma revisão integrativa (RI), que permite reunir informações de diversos estudos sobre um tema de maneira ordenada e sistemática. Para a condução do estudo, percorreram-se seis etapas: formulação da questão norteadora, busca na literatura dos estudos primários, extração de dados, avaliação dos estudos primários incluídos na revisão, análise e síntese dos resultados, e apresentação da revisão ${ }^{(13)}$. Para o rigor metodológico do estudo, observaram-se as recomendações do Checklist Prisma - Preferred Reporting Items for Systematic Reviews and Meta Analyses ${ }^{(14)}$.

O problema delimitou-se a partir do acrônimo PICo (Participantes, Fenômeno de Interesse e Contexto do Estudo) $)^{(15)}$, em que o Participante $(P)$ concerne a "cuidador de idoso"; o Fenômeno de Interesse (I) é referente á "tecnologia assistiva"; e o Contexto (C), refere-se a "estresse". Dessa forma, segue o seguinte questionamento: quais as evidências do uso de tecnologia assistiva na redução do estresse do cuidador de idoso?

A estratégia de busca dos estudos seguiu os critérios estabelecidos pelos manuais de cada base de dados. Sendo assim, os descritores controlados selecionados no MeSH (Medical Subject Headings), Emtree (Terminologia CINAHL), e DeCs (Descritores em Ciências da Saúde) e definidos conforme as palavras-chave, combinando-se com os operadores booleanos "AND" e "OR". A busca nas bases de dados ocorreu no período compreendido entre abril e maio de 2020 .

Utilizaram-se as bases e bancos de dados: Medical Literature Analysis and Retrieval System on-line (MEDLINE via PubMed ${ }^{\circledR}$ ), Web of Science ${ }^{T M}$, Cumulative Index to Nursing and Allied Health Literature (CINAHL-Ebsco), SciVerse Scopus (SCOPUS) e Biblioteca Virtual em Saúde (BVS). O acesso se deu por meio de uma instituição credenciada e liberada pelo Ministério da Educação, mediante os descritores controlados, não controlados e palavras-chave, como mostra o Quadro 1: 
Quadro 1 - Descritores controlados, descritores não controlados e palavras-chave selecionados nas bases de dados de acordo com acrônimo PICo.

\begin{tabular}{|c|c|c|c|}
\hline PICo & Descritores Controlados & Descritores não controlados & Palavras-chaves \\
\hline $\mathbf{P}$ & $\begin{array}{l}\text { Caregivers } \\
\text { Cuidadores }\end{array}$ & $\begin{array}{l}\text { Caregiver } \\
\text { carers } \\
\text { carer } \\
\text { caregivers } \\
\text { spouse caregivers } \\
\text { family caregivers }\end{array}$ & $\begin{array}{l}\text { idosos } \\
\text { pessoa idosa } \\
\text { pessoa de idade } \\
\text { pessoas idosas } \\
\text { pessoas de idade } \\
\text { população idosa } \\
\text { cuidador } \\
\text { cuidador familiar } \\
\text { cuidador de família } \\
\text { cuidadores cônjuges } \\
\text { cuidadores familiares } \\
\text { cuidadores de família } \\
\text { cônjuges cuidadores } \\
\text { familiar cuidador } \\
\text { familiares cuidadores } \\
\text { outro apoiador }\end{array}$ \\
\hline 1 & $\begin{array}{l}\text { self-help device } \\
\text { dispositivos de autoayuda } \\
\text { equipamentos de autoajuda }\end{array}$ & $\begin{array}{l}\text { device, self-help } \\
\text { self help device } \\
\text { assistive technology } \\
\text { technology, assistive } \\
\text { assistive device } \\
\text { device, assistive }\end{array}$ & $\begin{array}{l}\text { dispositivos assistivos } \\
\text { dispositivos de autoajuda } \\
\text { equipamentos assistivos } \\
\text { tecnologia assistiva }\end{array}$ \\
\hline Co & $\begin{array}{l}\text { occupational stress } \\
\text { estrés laboral } \\
\text { estresse ocupacional }\end{array}$ & $\begin{array}{l}\text { stress, occupational } \\
\text { job stress } \\
\text { stress, job } \\
\text { work-related stress } \\
\text { stress, work-related } \\
\text { work related stress } \\
\text { workplace stress } \\
\text { stress, workplace } \\
\text { workplace stress } \\
\text { stress, workplace } \\
\text { professional stress } \\
\text { stresses, professional } \\
\text { job-related stress } \\
\text { jobrelated stress }\end{array}$ & $\begin{array}{l}\text { estresse laboral } \\
\text { estresse profissional } \\
\text { estresse relacionado ao ambiente de trabalho } \\
\text { estresse relacionado ao trabalho } \\
\text { estresse relacionado à profissão } \\
\text { estresse do ambiente de trabalho } \\
\text { estresses laborais } \\
\text { estresses ocupacionais } \\
\text { estresses profissionais } \\
\text { estresses relacionados ao ambiente de trabalho } \\
\text { estresses relacionados à profissão } \\
\text { estresses do ambiente de trabalho }\end{array}$ \\
\hline
\end{tabular}

Consideraram-se elegíveis para esta revisão os estudos primários que respondessem à pergunta da pesquisa, disponíveis on-line nas bases de dados e sem restrição quanto ao idioma e ao recorte temporal até a conclusão da busca em maio de 2020, para a formulação do corpus e síntese de evidência. Excluiram-se os estudos de revisões, documentários, casos controle, teses, dissertações, trabalhos de conclusão de curso, estudos de reflexões, memoriais, documentários, editoriais, resenhas, cartas ao editor e artigos que não continham texto completo disponível gratuitamente.

Utilizou-se o gerenciador de referências EndNote para todas as bases de dados. Logo, procedeu-se à filtragem dos artigos com a realização da leitura de títulos e resumos, com exclusão de duplicatas e de estudos que não atendiam aos critérios de inclusão. Para a extração e síntese das informações dos estudos selecionados, aplicou-se um instrumento adaptado do formulário da Red de Enfermería en Salud Ocupacional (RedENSO Internacional) ${ }^{(16)}$. Por fim, extraíram-se as seguintes informações: base de dados, autores, título, ano, periódico, país do estudo, tipo de estudo, amostra, nível de evidência, objetivos, principais resultados e tecnologia assistiva. Utilizou-se o fluxograma para seleção dos estudos primários, conforme mostra a Figura 1. 


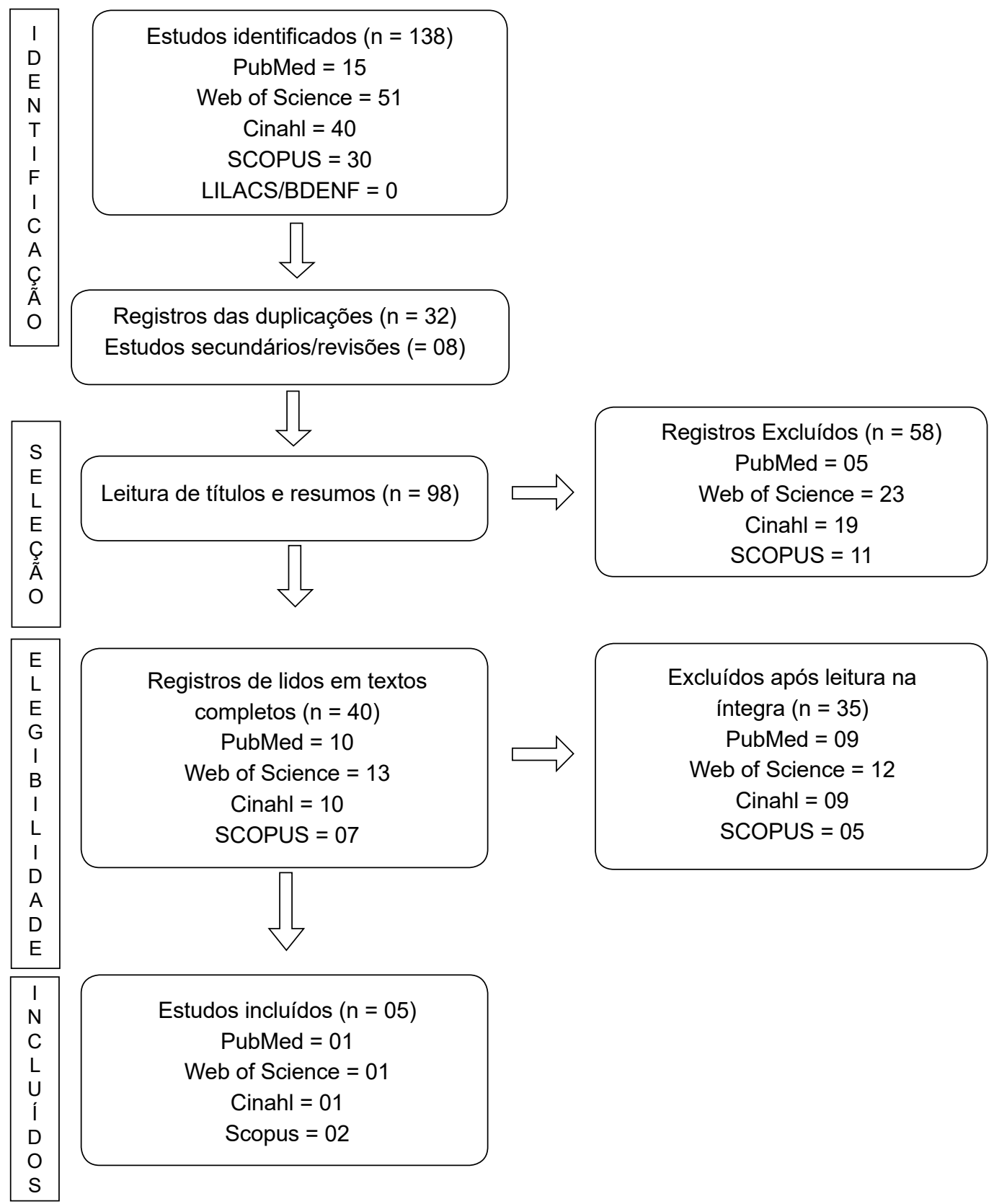

Figura 1 - Fluxograma de seleção dos estudos primários.

\section{RESULTADOS}

No que tange à etapa de análise de dados, após a estratégia de identificar os artigos elencados por letras e algarismos alfanuméricos " $\mathrm{A}_{\mathrm{n}}$ ", seguiu-se a análise por revisores independentes e, em caso de discordância, por uma juíza revisora.

A avaliação dos estudos apoiou-se na pirâmide de níveis de evidência que variam de I a VII, sendo: nível I metanálise ou revisões sistemáticas; nível II - ensaio clínico randomizado controlado; nível III - ensaio clínico sem randomização; nível IV - estudos de corte e de caso-controle; nível V - revisões sistemáticas de estudos descritivos e qualitativos; nível VI - estudos descritivos ou qualitativos; e nível VII - opinião de especialistas ${ }^{(17)}$.

Assim sendo, dos 138 artigos selecionados, apenas cinco estudos mencionavam o uso de tecnologia assistiva na redução do estresse do cuidador de idoso, conforme mostra o Quadro 2. Paralelamente, o Quadro 3 mostra autoria, ano da publicação, objetivo, principais resultados e tecnologia assistiva utilizada. 
Quadro 2 - Estudos selecionados segundo a base de dados, autores, título, ano, periódico, país do estudo, tipo de estudo, amostra e o nível de evidência.

\begin{tabular}{|c|c|c|c|c|c|}
\hline Estudo & $\begin{array}{l}\text { Base de } \\
\text { dados }\end{array}$ & Autores & Título & $\begin{array}{c}\text { Ano/ Periódico/ } \\
\text { País }\end{array}$ & $\begin{array}{l}\text { Tipo de estudo/amostra/ } \\
\text { Nível de evidência }\end{array}$ \\
\hline $\mathrm{A}_{1}$ & $\begin{array}{l}\text { WEB OF } \\
\text { SCIENCE }\end{array}$ & $\begin{array}{l}\text { LAU WM, CHAN } \\
\text { TY, SZETO SL }{ }^{(18)}\end{array}$ & $\begin{array}{l}\text { Effectiveness of a } \\
\text { home-based missing } \\
\text { incident prevention } \\
\text { program for } \\
\text { community-dwelling } \\
\text { elderly patients with } \\
\text { dementia }\end{array}$ & $\begin{array}{c}2019 \\
\text { International } \\
\text { Psychogeriatrics } \\
\text { China }\end{array}$ & 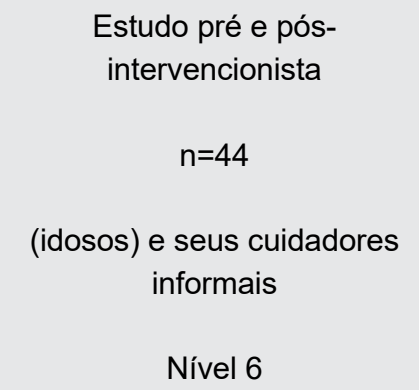 \\
\hline $\mathrm{A}_{2}$ & SCOPUS & $\begin{array}{l}\text { MORTENSONWB, } \\
\text { et al. } .^{(19)}\end{array}$ & $\begin{array}{c}\text { Caregivers' } \\
\text { experiences with the } \\
\text { selection and use of } \\
\text { assistive technology }\end{array}$ & $\begin{array}{l}2018 \\
\text { Disability and } \\
\text { Rehabilitation: } \\
\text { Assistive } \\
\text { Technology } \\
\text { Canadá }\end{array}$ & $\begin{array}{l}\text { Ensaio Clínico Randomizado } \\
\qquad \begin{array}{c}n=45 \\
\text { ( } \mathrm{n}=23 \text { experimental; } \\
\mathrm{n}=22 / \text { grupo de comparação) } \\
\text { Nível } 4\end{array}\end{array}$ \\
\hline $\mathrm{A}_{3}$ & PUBMED & $\begin{array}{l}\text { WANG RH, et } \\
\text { al. }{ }^{(20)}\end{array}$ & $\begin{array}{l}\text { Robots to assist } \\
\text { daily activities: views } \\
\text { of older adults with } \\
\text { Alzheimer's disease } \\
\text { and their caregivers }\end{array}$ & $\begin{array}{c}2017 \\
\text { Int Psychogeriatr } \\
\text { Canadá }\end{array}$ & 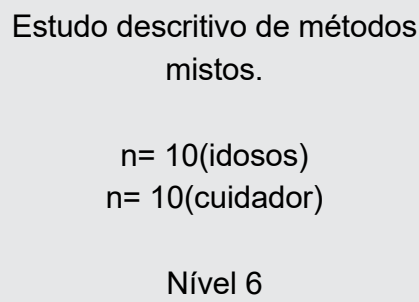 \\
\hline $\mathrm{A}_{4}$ & SCOPUS & $\begin{array}{l}\text { HATTINK BJJ, et } \\
\text { al. }^{(21)}\end{array}$ & $\begin{array}{l}\text { The electronic, } \\
\text { personalizable } \\
\text { Rosetta system } \\
\text { for dementia care: } \\
\text { exploring the } \\
\text { user-friendliness, } \\
\text { usefulness, and } \\
\text { impact }\end{array}$ & $\begin{array}{l}2016 \\
\text { Disability and } \\
\text { Rehabilitation: } \\
\text { Assistive } \\
\text { Technology. } \\
\text { Holanda, } \\
\text { Alemanha e } \\
\text { Bélgica }\end{array}$ & $\begin{array}{c}\text { Estudo de avaliação } \\
\text { exploratória } \\
\mathrm{n}=42 \\
\text { (19 na Holanda, } 11 \text { na } \\
\text { Alemanha e } 12 \text { na Bélgica) } \\
\text { e } \mathrm{n}=32 \text { de seus cuidadores } \\
\text { informais. } \\
\text { Nível } 6\end{array}$ \\
\hline $\mathrm{A}_{5}$ & CINAHL & $\begin{array}{c}\text { McKENZIE B, et } \\
\text { al.(22) }\end{array}$ & $\begin{array}{l}\text { Safe Home } \\
\text { Program: } \text { A Suite } \\
\text { of Technologies to } \\
\text { Support Extended } \\
\text { Home Care of } \\
\text { persons with } \\
\text { dementia }\end{array}$ & $\begin{array}{l}2013 \\
\text { American Journal } \\
\text { of Alzheimer's } \\
\text { Disease \& Other } \\
\text { Dementias } \\
\text { Estados Unidos }\end{array}$ & $\begin{array}{l}\text { Transversal } \\
\qquad \text { n=60 } \\
\text { Nível } 6\end{array}$ \\
\hline
\end{tabular}


Quadro 3 - Estudos primários selecionados segundo as tecnologias assistivas utilizadas para redução do estresse em cuidadores de idosos.

\begin{tabular}{|c|c|c|c|c|}
\hline Estudo & Autores / Ano & Objetivo & Principais Resultados & $\begin{array}{c}\text { Tecnologia } \\
\text { Assistiva }\end{array}$ \\
\hline A1 & $\begin{array}{l}\text { LAU WM, } \\
\text { CHAN TY, } \\
\text { SZETO SL, } \\
2019^{(18)}\end{array}$ & $\begin{array}{l}\text { Examinar a eficácia de um } \\
\text { programa abrangente de } \\
\text { prevenção de incidentes } \\
\text { perdidos em casa, a redução } \\
\text { do número de pacientes } \\
\text { desaparecidos, incidentes } \\
\text { perdidos, tempo de procura e } \\
\text { estresse dos cuidadores em } \\
\text { comunidades de moradores } \\
\text { com demência. }\end{array}$ & $\begin{array}{l}\text { O número total de pacientes e episódios com } \\
\text { perda, estresse dos cuidadores e tempo médio } \\
\text { de pesquisa apresentaram-se significativamente } \\
\text { reduzidos. Havia seis componentes de serviço } \\
\text { nesse programa domiciliar e a maioria dos } \\
\text { itens se fez sob medida, de acordo com as } \\
\text { necessidades dos pacientes e o conhecimento } \\
\text { dos cuidadores. O estudo demonstrou que uma } \\
\text { intervenção multidimensional personalizada e } \\
\text { domiciliar fornecida por um terapeuta ocupacional } \\
\text { pode reduzir episódios ausentes e o estresse dos } \\
\text { cuidadores entre pacientes com demência. }\end{array}$ & $\begin{array}{l}\text { Auxiliares de } \\
\text { memória e } \\
\text { Dispositivos de } \\
\text { rastreamento. }\end{array}$ \\
\hline $\mathrm{A}_{2}$ & $\begin{array}{l}\text { MORTENSON, } \\
\text { WB, et al. } \\
2018^{(19)}\end{array}$ & $\begin{array}{l}\text { Examinar os efeitos de um } \\
\text { cuidador inclusivo por } 6 \\
\text { semanas. }\end{array}$ & $\begin{array}{l}\text { A autonomia funcional do usuário com tecnologia } \\
\text { assistiva diminuiu, enquanto a carga do cuidador } \\
\text { mostrou-se reduzida. Essas descobertas } \\
\text { ocorreram apesar de a intervenção experimental } \\
\text { ter como alvo atividades mais problemáticas, } \\
\text { fornecer mais TA e incluir mais visitas de } \\
\text { terapeutas ocupacionais. }\end{array}$ & $\begin{array}{l}\text { Bengalas, } \\
\text { andadores, cadeiras } \\
\text { de rodas, barras } \\
\text { de apoio, assentos } \\
\text { sanitários elevados } \\
\text { e bancos de banho. }\end{array}$ \\
\hline $\mathrm{A}_{3}$ & $\begin{array}{l}\text { WANG RH, } \\
\text { et al. 2017(20) }\end{array}$ & $\begin{array}{l}\text { Permitir que idosos } \\
\text { com demência realizem } \\
\text { atividades diárias com maior } \\
\text { independência e fornecer } \\
\text { apoio aos cuidadores. }\end{array}$ & $\begin{array}{l}\text { Os cuidadores identificaram inúmeras } \\
\text { oportunidades e apresentaram-se receptivos } \\
\text { aos robôs assistivos, obtendo consequências } \\
\text { positivas em cenários do cuidado. Assim, houve } \\
\text { diminuição da frustração, estresse e tensão no } \\
\text { relacionamento e aumento da interação social } \\
\text { por meio do robô. Como consequência negativa, } \\
\text { houve a diminuição da interação entre idosos e } \\
\text { cuidadores. }\end{array}$ & $\begin{array}{l}\text { Robô de Alerta } \\
\text { Assistencial } \\
\text { (movimento, } \\
\text { comunicação e } \\
\text { estímulo). }\end{array}$ \\
\hline $\mathrm{A}_{4}$ & $\begin{array}{l}\text { HATTINK BJJ, } \\
\text { et al. } 2016^{(21)}\end{array}$ & $\begin{array}{l}\text { Integrar três sistemas de } \\
\text { tecnologia assistencial } \\
\text { (TA) desenvolvidos em } \\
\text { um sistema modular e } \\
\text { multifuncional, que poderá } \\
\text { apoiar pessoas com } \\
\text { demência e prestadores de } \\
\text { cuidados durante o curso da } \\
\text { patologia. }\end{array}$ & $\begin{array}{l}\text { O sistema utilizado obteve um impacto na } \\
\text { vida dos usuários. Sentiram-se seguros e } \\
\text { confortáveis. Especialmente com o subsistema } \\
\text { com função de detecção de queda e suporte de } \\
\text { memória através da tela de toque e do dispositivo } \\
\text { móvel. Os usuários do sistema classificaram } \\
\text { sua facilidade de uso como baixa. As pessoas } \\
\text { com demência e seus cuidadores informais } \\
\text { consideraram o sistema útil para ajudar a manter } \\
\text { a independência, lembrando as pessoas com } \\
\text { demência de compromissos, que os cuidadores } \\
\text { informais podem esquecer. As pessoas com } \\
\text { demência e cuidadores relataram que a TA } \\
\text { pode ajudar a promover a segurança e reduzir a } \\
\text { sobrecarga e o estresse. }\end{array}$ & $\begin{array}{l}\text { Software - } \\
\text { Sistema Rosetta } \\
\text { - três subsistemas: } \\
\text { navegador do dia } \\
\text { do idoso, software } \\
\text { de sistema de } \\
\text { detecção precoce } \\
\text { e sistema de } \\
\text { vigilância autônoma } \\
\text { - consciência } \\
\text { e prevenção } \\
\text { avançadas. }\end{array}$ \\
\hline$A_{5}$ & $\begin{array}{c}\text { MCKENZIE B, } \\
\text { et al. 2013(22) }\end{array}$ & $\begin{array}{l}\text { Relatar a implementação/ } \\
\text { adoção do Programa } \\
\text { Casa Segura para apoiar } \\
\text { cuidadores de pessoas com } \\
\text { demência em vigilância } \\
\text { contínua, prestação de } \\
\text { cuidados, prevenção } \\
\text { de lesões e melhoria da } \\
\text { segurança em casa. }\end{array}$ & $\begin{array}{l}\text { As tecnologias emitidas pelo programa } \\
\text { aumentaram a sensação de segurança e } \\
\text { melhoraram o estresse da carga associado } \\
\text { ao atendimento de pessoas com demência e } \\
\text { ajudaram a garantir que a pessoa com demência } \\
\text { permanecesse segura em casa. }\end{array}$ & $\begin{array}{l}\text { Programa Safe } \\
\text { Home. }\end{array}$ \\
\hline
\end{tabular}

Os cinco estudos primários selecionados estão no idioma inglês (100\%), cujos países de origem contam-se como Canadá, dois (40\%); Estados Unidos, um (20\%); China, um (20\%). Além disso, apresentou-se outro estudo realizado concomitantemente entre três países: Holanda, Alemanha e Bélgica, um (20\%). Com relação ao período 
de publicação, observou-se que quatro (80\%) tiveram suas publicações entre os anos 2016 a 2019 e um (20\%) ano 2013. Já quanto ao nível de evidências, encontraram-se quatro (80\%) nível VI e um (20\%) nível IV.

Os estudos selecionados mostram que a usabilidade de tecnologias assistivas são capazes de facilitar o trabalho realizado por cuidadores de idosos dependentes e/ou portadores de doença senil. Com isso identificaram-se tecnologias assistivas de diferentes tipos, como programas de computadores (softwares), uso de robôs e programas domiciliares.

\section{DISCUSSÃO}

O uso de Tecnologia Assistida (TA) vem modificando o modo de vida de idosos dependentes e seus cuidadores, pois melhoram o bem-estar físico e emocional da díade, trazendo maior independência para idosos e cuidadores ${ }^{(18-22)}$.

Evidenciaram-se os benefícios relacionados aos cuidadores de idosos, no qual em sua grande maioria ocorreram aos cuidadores de idosos com demência senil. Nesse sentido, os estudos ${ }^{(18,20-22)}$ demonstraram que as tecnologias assistivas são capazes de amenizar a sobrecarga de trabalho exercida por cuidadores, por meio de programas domiciliares, resultando em melhoria na qualidade de vida, por promoverem segurança e confiança.

Esse achado está em concordância com outro estudo que evidencia a aplicabilidade de TA na melhoria da qualidade de vida de idosos com demência e seus cuidadores, pois auxiliam os cuidadores a executarem ações de cuidado de maneira mais promissora(23).

Outros estudos chegaram à mesma conclusão no que tange ao potencial das tecnologias assistivas como suporte ao cuidado de idosos com alguma patologia relacionada ao sistema nervoso, como na demência senil e alzheimer. E sobre isso, apurou-se que cuidadores informais obtiveram como benefícios a diminuição da sobrecarga de trabalho ${ }^{(18-22,24,25)}$.

Constatou-se que associar o uso de TA na promoção do cuidado ao idoso dependente pode ser vista como uma necessidade de prevenir agravos à saúde do cuidador, pois sabe-se que o cuidador, na maioria das vezes, é um familiar próximo, que precisa conciliar outros afazeres a esta nova função, quase sempre exercida sem descanso, ou seja, todos os dias, durante as 24 horas a depender do grau de dependência do idoso, podendo ocasionar sentimento de frustração e estresse para esse cuidador. Verificou-se que as tecnologias assistivas são ferramentas inovadoras que podem ser capazes de promover a qualidade de vida do idoso e do cuidador formal ou informal, podendo ser classificadas em tecnologias simples (andador, bengala, bastão, elevação de acento sanitário) e tecnologias complexas (sensores de queda, sensores de ocupação de cama, sensores de iluminação, casa monitorada, alarmes). A diferenciação entre os tipos se dá na necessidade ou não de um aparato tecnológico avançado; as tecnologias assistivas simples não requerem esses aparatos, enquanto as complexas requerem ${ }^{(26)}$.

Ainda que as tecnologias assistivas sejam utilizadas na maior parte pelos idosos dependentes ou em seu benefício mediante as limitações decorrentes de diversos fatores associados à idade avançada, permite-se aos cuidadores, de forma direta ou indireta, obter benefícios durante o auxílio na prestação de cuidados ao idoso dependente. Nesta revisão, identificaram-se tecnologias assistivas de diferentes tipos, como programas de computadores (softwares), uso de robôs e programas de cuidado domiciliar que contavam com ferramentas específicas simples (bengalas, andadores, cadeiras de rodas, barras de apoio, assentos sanitários elevados, bancos de banho) e complexas (dispositivos de rastreamento, sensores de movimento, câmeras de segurança, sistemas de alerta domiciliar, entre outros) $)^{(18-22)}$.

Desta forma, mediante a análise dos estudos ${ }^{(18-22)}$, houve a redução do estresse como benefício primário da utilização de TA. Além disso, identificaram-se também outros benefícios, como diminuição de carga de trabalho, diminuição do "fardo" do cuidador informal e geração de sentimentos de segurança, tanto nos cuidadores informais quanto nos idosos dependentes.

Apresentou-se outro benefício na utilização de TA em relação à promoção da saúde do idoso e do cuidador. Para o idoso houve manutenção da autonomia, promoção do conforto e otimização do tempo perdido em atividades diárias. Para o cuidador houve redução da frustração em relação ao cuidado dispensado ao idoso. Dessa forma, considerou-se que o uso de TA no cuidado domiciliar ao idoso dependente é de primordial importância já que promove a saúde da díade ${ }^{(18-22,27)}$. Verificou-se que os recursos de TA são capazes de apoiar e facilitar o trabalho da equipe de cuidado e dos cuidadores familiares se os dispositivos tiverem utilidade e usabilidade suficientes, como fácil comissionamento, manutenção e personalização às necessidades específicas de usabilidade do usuário ${ }^{(28)}$.

Nesta revisão, um estudo apontou que os recursos de Tecnologia Assistida podem reduzir a interação entre o idoso e o cuidador, principalmente quando essas tecnologias são complexas, como por exemplo a utilização dos 
robôs ${ }^{(20)}$. Portanto, deve-se procurar manter o equilíbrio entre a inserção de maquinário tecnológico e a humanização do cuidado. Com efeito, as tecnologias duras devem ser implementadas com as tecnologias leves, vislumbrando o cuidado humanizado e a integralidade do ser idoso cuidado como estratégia de promoção da saúde ${ }^{(29)}$ e redução do estresse em cuidadores de idosos.

Entende-se que as TA complexas podem apresentar facilidades e dificuldades na sua utilização, tanto para o idoso quanto para seu cuidador. No entanto, julga-se necessário que haja orientação, treinamento, acompanhamento, supervisão, acessibilidade e determinação para seu uso. Constatou-se, em um dos estudos, que as tecnologias assistivas complexas para o cuidado das pessoas idosas dependentes e com demência, utilizou-se de dispositivos de rastreamento por meio de programas de internet, serviços de GPS e aparelho de telefone celular. Estes dispositivos localizavam objetos perdidos dentro de casa, como também rastreavam idosos desaparecidos. Como resultado houve uma redução de desperdício de tempo perdido e melhora na qualidade de vida da díade, pois as tecnologias assistivas utilizadas ajudaram os cuidadores a desenvolver estratégias preventivas no enfrentamento de doenças no dia a dia. Além disso, em outro estudo demonstrou-se que um programa de intervenção personalizada reduz o estresse do cuidador ${ }^{(18)}$.

Contudo, é importante pontuar que os efeitos da demência e de outras doenças que acometem idosos, podem comprometer a saúde física, mental e social, e, sobretudo, sua independência para o autocuidado. Ademais, outro fator a ser pontuado é a possibilidade de também ocorrer um comprometimento na saúde e no bem-estar do cuidador, por exigir atenção física e mental para atuar no cuidado, pois requer atenção e dedicação especial ${ }^{(30)}$.

Pôde-se observar que os estudos envolvendo os recursos de TA estão presentes principalmente em países desenvolvidos, assim como a acessibilidade a tais tecnologias por idosos e seus cuidadores. Contudo, a utilização de uma tecnologia pode auxiliar o idoso a desenvolver suas atividades diárias com certa independência, contribuindo para o bem-estar e a qualidade de vida da díade. Corrobora-se que os recursos de TA funcionam como auxílio para propiciar o desenvolvimento de habilidades comprometidas, possibilitando a funcionalidade desejada que encontra-se impossibilitada em decorrência de deficiência ou pelo envelhecimento ${ }^{(10)}$.

De certo, a TA é uma das ferramentas que têm sido utilizadas com sucesso para manter a independência dos idosos, apresentando informações sobre quais surgem como necessidades de TA para o dia a dia, contribuindo, então, para aumentar a efetividade do fornecimento de TA para essa população. Contudo, novas políticas precisam ser desenvolvidas para defender o aumento da cobertura de saúde de dispositivos de TA de baixa tecnologia que possam, então, apresentar benefícios para pessoas mais idosas e seus cuidadores ${ }^{(31)}$.

As tecnologias ainda estão distantes da realidade da maior parte da população diante do perfil socioeconômico da população brasileira ${ }^{(12)}$. Desta forma, entende-se que uma política voltada para a acessibilidade, o treinamento em educação em saúde e o acompanhamento supervisionado poderiam contribuir para enaltecer a qualidade de vida dos cuidadores de idosos dependentes. As contribuições deste estudo são importantes para o preenchimento de uma lacuna na promoção da saúde do cuidador de idoso dependente, no entanto, é necessário que haja outros estudos que possam mostrar maiores evidências sobre a redução do estresse no cuidador com o uso de TA, a fim de melhorar a atenção a saúde do cuidador, permitindo reduzir danos à sua saúde mental.

E para finalizar, como limitação para o presente estudo, destaca-se a escassez de publicações que respondessem à questão da pesquisa, a fragilidade metodológica e o baixo nível de evidência dos artigos disponíveis na literatura.

\section{CONCLUSÃO}

Os artigos selecionados para esta revisão mostraram que as tecnologias assistivas melhoram o estresse associado à sobrecarga dos cuidadores de idosos, possibilitando melhoria em sua qualidade de vida, com redução das tensões, estresse e frustrações. No entanto, as tecnologias assistivas precisam estar acessíveis para serem utilizadas, de modo a proporcionar mudanças efetivas na promoção da saúde do cuidador.

\section{CONFLITOS DE INTERESSE}

Os autores afirmam que não houve conflitos de interesses na execução desta pesquisa.

\section{CONTRIBUIÇÕES}

Gerarlene Ponte Guimarães Santos, Cleidiane Maria Sales de Brito, Rayla Maria Pontes Guimarães Costa contribuíram com a elaboração e delineamento do estudo; a aquisição, análise e interpretação de dados; e a 
redação e/ou revisão do manuscrito. Herla Maria Furtado Jorge, Ana Roberta Vilarouca da Silva, Maria do Livramento Fortes Figueiredo, Márcia Teles de Oliveira Gouveia, Márcia Astrês Fernandes contribuíram com a redação e/ou revisão do manuscrito. Todos os autores aprovaram a versão final a ser publicada e são responsáveis por seu conteúdo.

\section{REFERENNCIAS}

1. Kreling NH. Envelhecimento e inserção do idoso no mercado de trabalho, na Região Metropolitana de Porto Alegre. Indic Econ Fee [Internet]. 2016 [acesso em 2016 Mar 7];43(3):141-54. Disponível em: https://revistas. fee.tche.br/index.php/indicadores/article/view/3666

2. Instituto Brasileiro de Geografia e Estatística. Pesquisa Nacional por Amostra de Domicílios Contínua: características gerais dos domicílios e dos moradores 2018 [Internet]. Rio de Janeiro: IBGE, 2019 [acesso em 2016 Maio 6]. Disponível em: https://biblioteca.ibge.gov.br/visualizacao/livros/liv101654_informativo.pdf

3. Organização Pan-Americana de Saúde. Search for folha informativa envelhecimento e saúde Brasil [Internet]. Brasília: OPAS, 2018 [acesso em 2021 Maio 6]. Disponível em: https://www.paho.org/bra/index. php?option=com_content $\&$ view=article\&id=5661: folha-informativa-envelhecimento-e-saude \&ltemid $=820$

4. Gomes NP, Pedreira LC, Gomes NP, Fonseca EOS, Reis LA, Santos AA. Health-related consequences of caring for dependent relatives in older adult caregivers. Rev Esc Enferm USP [Internet]. 2019 [acesso em 2021 maio 9];53:e03446. Disponível em: http://www.scielo.br/scielo.php?script=sci_arttext\&pid=S008062342019000100418\&Ing=en doi: https://doi.org/10.1590/s1980-220x2018002303446

5. Assenheimer A, Brum ZP. Dificuldades enfrentadas pelo cuidador de idoso domiciliar. RICSB [Internet]. 2019 [acesso em 2020 Maio 17];3(2):3-12.

6. Delalibera M, Barbosa A, Leal I. Circunstâncias e consequências do cuidar: caracterização do cuidador familiar em cuidados paliativos. Ciênc Saúde Colet [Internet]. 2018 [acesso em 2021 Abr 30];23(4):1105-17. Disponível em: http://www.scielo.br/scielo.php?script=sci_arttext\&pid=S141381232018000401105\&Ing=en doi: 10.1590/1413-81232018234.12902016

7. Kobayasi DY, Rodrigues PR, Fhon SJ, Silva LM, Souza AC, Chayamiti CE. Sobrecarga, rede de apoio social e estresse emocional do cuidador do idoso. Av Enferm [Internet]. 2019 [acesso em 2021 Abr 30];37(2):140-8. doi: 10.15446/av.enferm.v37n2.73044

8. Bersch R. Introdução à tecnologia assistiva. Porto Alegre: Assistiva Tecnologia e Educação; 2017.

9. Maia JC, Coutinho JF, Sousa CR, Barbosa RG, Mota FR, Marques MB, et al. Tecnologias assistivas para idosos com demência: revisão sistemática. Acta Paul Enferm [Internet]. 2018 Dez [acesso em 2021 Maio 4];31(6):651-8. Disponível em: http://www.scielo.br/scielo.php?script=sci_arttext\&pid=S010321002018000600651\&lng=en doi: 10.1590/1982-0194201800089

10. Leite ES, Pimenta CJL, Costa MS, Oliveira FB, Moreira MASP, Silva AO. Assistive technology and active aging according to professionals working in community groups. Rev Esc Enferm USP [Internet]. 2018 [acesso em 2021 Maio 4];52:e03355. Disponivel em: http://www.scielo.br/scielo.php?script=sci_arttext\&pid=S008062342018000100441\&Ing=pt doi: 10.1590/s1980-220x2017030903355

11. Williams K, Blyler D, Vidoni ED, Shaw C, Wurth J, Seabold D, et al. A randomized trial using telehealth technology to link caregivers with dementia care experts for in-home caregiving support: FamTechCare protocol. Res Nurs Health [Internet]. 2018 Jun [acesso em 2021 Maio 4];41(3):219-27. Disponível em: https:// pubmed.ncbi.nlm.nih.gov/29504666 doi: 10.1002/nur.21869

12. Coimbra TM, Ezequiel CT, Moreira DS, Morita MPA, Castiglioni L, Bianchin MA. Comunicação Alternativa Ampliada na Esclerose Lateral Amiotrófica: A Tecnologia a Favor da Reabilitação. Arqu Ciênc Saúde [Internet]. 2018 [acesso em 2021 Maio 4];25(3):22-6. Disponível em: https://www.cienciasdasaude.famerp.br/ index.php/racs/article/view/1054 doi: 10.17696/2318-3691.25.3.2018.1054

13. Whittemore R, Chao A, Jang M, Minges KE, Park C. Methods for knowledge synthesis: an overview. Heart Lung. 2014;5(43):453-61.

14. Liberati A, Altman DG, Tetzlaff J, Mulrow C, Gøtzsche PC, loannidis JPA, et al. The PRISMA statement 
for reporting systematic reviews and meta-analyses of studies that evaluate health care interventions: explanation and elaboration. PloS Med [Internet]. 2009 [acesso em 2020 Maio 17];7(3). Disponível em: https://journals. plos.org/plosmedicine/article?id=10.1371/journal.pmed. 1000100

15. Lockwood C, Porrit K, Munn Z, Rittenmeyer L, Salmond S, Bjerrum M, et al. Systematic reviews of qualitative evidence. In: Aromataris E, Munn Z, editores. JBI Manual for Evidence Synthesis [Internet]: JBI; 2020 [acesso em 2020 Maio 17] Disponível em: https://synthesismanual.jbi.global. doi: https://doi.org/10.46658/ JBIMES-20-03

16. Marziale MH. Instrumento para recolección de datos revisión integrativa [Internet]. São Paulo: RedENSO; 2015 [acesso em 2020 Jun 25]. Disponível em: http://gruposdepesquisa.eerp.usp.br/sites/redenso/wpcontent/uploads/sites/9/2019/09/Instrumiento_revision_literaatura_RedENSO_2015.pdf

17. Melnyk BM, Fineout-Overholt E. Evidence-based practice in nursing \& healthcare: a guide to best practice. Philadelphia: Lippincott Willians Wilkins; 2005. Making the case for evidence-based practice; p. 3-24.

18. Lau WM, Chan TY, Szeto SL. Effectiveness of a home-based missing incident prevention program for community-dwelling elderly patients with dementia. Int Psychogeriatr [Internet]. 2019 [acesso em 2020 Jul 3];31(1):91-9. Disponível em: https://pubmed.ncbi.nlm.nih.gov/29720290/

19. Mortenson WB, Pysklywec A, Fuhrer MJ, Jutai JW, Plante M, Demers L. Caregivers' experiences with the selection and use of assistive technology. Disabil Rehabil Assist Technol [Internet]. 2018 [acesso em 2020 Jul 3];13(6):562-7. Disponível em: https://pubmed.ncbi.nlm.nih.gov/28768438/

20. Wang RH, Sudhama A, Begum M, Huq R, Mihailidis A. Robots to assist daily activities: views of older adults with Alzheimer's disease and their caregivers. Int Psychogeriatr [Internet]. 2017 [acesso em $2020 \mathrm{Jul}$ 3];29(1):67-79. Disponível em: https://pubmed.ncbi.nlm.nih.gov/27660047/

21. Hattink BJJ, Meiland FJM, Overmars-Marx T, Boer M, Ebben PWG, Blanken MV. The electronic, personalizable Rosetta system for dementia care: exploring the user-friendliness, usefulness and impact. Disabil Rehabil Assist Technol [Internet]. 2016 [acesso em 2020 Jul 3];11(1):61-71. Disponível em: https:// pubmed.ncbi.nlm.nih.gov/24989993/

22. McKenzie B, Bowen ME, Keys K, Bulat T. Safe home program: a suite of technologies to support extended home care of persons with dementia. Am J Alzheimers Dis Other Demen [Internet]. 2013 [acesso em 2020 Jul 3];28(4):348-54. Disponível em: https://pubmed.ncbi.nlm.nih.gov/23677733/

23. Maia JC, Coutinho JFV, Sousa CR, Barbosa RGB, Mota FRN, Marques MB, et al. Assistive technologies for demented elderly: a systematic review. Acta Paul Enferm [Internet]. 2018 [acesso em 2020 Jul 3];31(6):651-8. Disponível em: http://www.scielo.br/scielo.php?script=sci_arttext\&pid=S0103-21002018000600651\&lng=en\&n $\mathrm{rm}=$ iso

24. lenca M, Jotterand F, Elger B, Caon M, Pappagallo AS, Kressig RW, et al. Intelligent assistive technology for alzheimer's disease and other dementias: a systematic review. J Alzheimers Dis [Internet]. 2017 [acesso em 2020 Jul 3];4(56):1301-40. Disponível em: https://www.researchgate.net/publication/313461148_Intelligent_ Assistive_Technology_for_Alzheimer's_Disease_and_Other_Dementias_A_Systematic_Review

25. Carmo EG, Zazzetta MS, Fuzaro G Jr, Micali PN, Moraes PF, Costa JLR. A utilização de tecnologias assistivas por idosos com Doença de Alzheimer. Rev Kairós Gerontol [Internet]. 2015 [acesso em 2020 Jul 3];18(4):311-36. Disponível em: https://revistas. pucsp.br/kairos/article/view/29507/20572

26. Gibson G, Newton L, Pritchard G, Finch T, Brittain K, Robinson L. The provision of assistive technology products and services for people with dementia in the United Kingdom. Dementia [Internet]. 2014 [acesso em 2020 Jul 3];15(4):681-701. doi: 10.1177/1471301214532643

27. letrzak E, Cotea C, Pullman S. Does smart home technology prevent falls in community-dwelling older adults: a literature review. Inform Prim Care [Internet]. 2017 [acesso em 2020 Jul 3];21(3):105-12. Disponível em: https://pubmed.ncbi.nlm.nih.gov/25207613/

28. Sriram V, Jenkinson C, Peters M. Informal carers' experience of assistive technology use in dementia care at home: a systematic review. BMC Geriatrics [Internet]. 2019 [acesso em 2020 Jul 3];19(1):160. Disponível em: https://bmcgeriatr.biomedcentral.com/articles/10.1186/s12877-019-1169-0 
29. Araújo SNM, Santiago RF, Barbosa CNS, Figueiredo MLF, Andrade EMLR, Nery IS. Tecnologias voltadas para o cuidado ao idoso em serviços de saúde: uma revisão integrativa. Enferm Global [Internet]. 2017 [acesso em 2020 Maio 17];(46):579-95. doi: 10.6018/eglobal.16.2.247241

30. Faieta J, Sheehan J, Digiovine C. Mhealth interventions to improve health and quality of life related outcomes for informal dementia caregivers: a scoping review. Assist Technol [Internet]. 2021 Mar 5; [Epub ahead of print]. Disponível em: https://pubmed.ncbi.nlm.nih.gov/33006526/ doi: 10.1080/10400435.2020.1829174

31. Orellano-Colón EM, Rivero-Méndez M, Lizama M, Jutai JW. Assistive technology unmet needs of independent living older Hispanics with functional limitations. Disabil Rehabil Assist Technol [Internet]. 2018 [acesso em 2020 Jul 3];13(2):194-200. Disponível em: https://pubmed.ncbi.nlm.nih.gov/28326905/

\section{Endereço para correspondência: \\ Gerarlene Ponte Guimarães Santos \\ Avenida Nossa Senhora de Fátima, S/N \\ Bairro: Fátima \\ CEP 64202-220 - Parnaíba - PI - Brasil \\ E-mail: gerarleneg@gmail.com}

Como citar: Santos GPG, Brito CMS, Costa RMPG, Jorge HMF, Silva ARV, Gouveia MTO, et al. Tecnologia assistiva e a redução do estresse em cuidadores de idosos: revisão integrativa. Rev Bras Promoç Saúde. 2021;34:11488. 